\section{Microintervenciones didácticas \\ para la alfabetización de adultos en contextos adversos}

\author{
Micaela Dallia \\ micaeladallia@hotmail.com
}

Mariana Oggioni

marianaoggioni@live.com

Natasha Rudzki

nataa.r@hotmail.com

Lorena Sánchez

loresanchez92@hotmail.com

Alumnas de la Facultad de

Nacional del Litoral, Argentina.
Humanidades y Ciencias. Universidad

\section{Cintia Carrió}

Docente investigadora de la Facultad de Humanidades y Ciencias. Universidad Nacional del Litoral Consejo Nacional de Investigaciones Científicas y Tecnológicas (CONICET) ccarrio@unl.edu.ar

\section{Resumen}

En este artículo se relata la experiencia de aprendizaje mutuo entre un grupo de voluntarios-estudiantes avanzados de la carrera de Letras y un grupo de adultos de una comunidad aborigen. El objetivo del proyecto apuntó a la alfabetización inicial de adultos de la comunidad, quienes no contaban con conocimientos previos o con experiencias escolares mínimas. Con claros propósitos de interrelación, aprendizaje y reconocimientos de las diferencias culturales, se abordó el trabajo desde una metodología de alfabetización que fue mutando y adaptándose a las necesidades del medio, lo que pone de manifiesto la necesidad de continuo diálogo y retroalimentación entre las tareas de extensión e investigación. El objetivo concreto, planteado desde el equipo, fue lograr avances básicos en la escritura, en la lectura y la posibilidad de rubricar la propia identidad (el propio yo). El desafío consistió en readaptar las expectativas y sobrellevar los obstáculos sociales, organizativos y epistemológicos. Estos últimos, especialmente, resaltando las tensiones entre formación y actuación de los conocimientos.

Palabras-clave

- Escritura

- Lectura

- Decisiones didácticas

- Minorías étnicas

- Investigación y extensión
Integración de la docencia y la extensión /

Intervenciones

RECEPCIÓN: 24/06/16

ACEPTACIÓN FINAL: 10/10/16

\section{Resumo}

Neste artigo se relata uma experiência de aprendizagem mútua entre um grupo de voluntários-estudantes avançados da faculdade de Letras e um grupo de adultos de uma comunidade indígena. $\mathrm{O}$ objetivo do projeto visou à alfabetização inicial de adultos sem conhecimentos prévios ou experiências escolares anteriores. $\mathrm{O}$ trabalho se abordou a partir de uma metodologia de alfabetização que foi mudando e se adaptando às necessidades do meio, favorecendo a interrelação, aprendizagem e reconhecimento das diferenças culturais. Isto evidencia a necessidade de um diálogo contínuo e feedback entre as tarefas de extensão e pesquisa.

O objetivo concreto, traçado pela equipe, foi conseguir avanços básicos na escrita, na leitura e a possibilidade de afirmar a própria identidade (o próprio eu). O desafio consistiu em readaptar as expectativas e superar os obstáculos sociais, organizacionais e epistemológicos. Estes últimos, especialmente, ressaltando as tensões entre formação e desempenho do conhecimento.
Palavras-chave

- Escrita

- Leitura

- Decisões didáticas

- Minorias étnicas

- Pesquisa e extensão
Para citación de este artículo

Dallia, M.; Oggioni, M.; Rudzki, N.; Sánchez, L. y Carrió, C. (2016). Microintervenciones didácticas para la alfabetización de adultos en contextos adversos. En Revista $+E$ versión digital, (6), pp. 370-375. Santa Fe, Argentina: Ediciones UNL. 
"Poder leer y escribirle cartas a mi hijo". Rosa

\section{Introducción}

Toda experiencia de interacción con el medio se constituye, de una forma u otra, en una instancia de aprendizaje. Ahora bien, en aquellos casos en que los conocimientos académicos son puestos en abismo constantemente suponen en un desafío.

En este artículo se relata la experiencia de aprendizaje mutuo entre un grupo de voluntarios-estudiantes avanzados de la carrera Profesorado de Letras (FHUC-UNL) y un grupo de adultos de una comunidad aborigen de la provincia de Santa Fe. ${ }^{1}$ Se detalla una experiencia de microintervención didáctica para la alfabetización de adultos, entendiendo por "microintervención" una planificación pautada y controlada para un contexto concreto, una propuesta focalizada (ver. "nano-intervención" en Gerbaudo, 2016, y las referencias allí incluidas). Aquí entonces se busca dar cuenta de la experiencia, de los conflictos de diferente índole que fue necesario superar para llevar adelante el trabajo planificado. Asimismo, se da cuenta de los aprendizajes adquiridos con esta experiencia y de las tensiones que se visibilizaron al poner en relación lo aprendido en el ámbito universitario y lo demandado por el medio.

\section{Contexto y actores 1}

La comunidad de Campo San José pertenece al grupo aborigen mocoví. Los habitantes de la localidad comenzaron hace unos pocos años a ejercer sus derechos sobre estas tierras, para lo cual la residencia permanente fue, sin dudas, la decisión más radical y a la vez más dura. Esto así dado que en la localidad no están garantizadas las necesidades básicas de luz y agua sino parcialmente o de manera interexcluyente.

Las condiciones, aún precarias aunque incipientes, de organización urbano-rural traen como resultado serias complejidades para acceder a los diferentes niveles de escolarización. En este sentido, las familias residentes presentan una situación en la que hombres y mujeres trabajan en quintas ${ }^{2}$ propias, o bien los hombres (principalmente) realizan trabajos fuera de la comunidad. Las condiciones laborales impactan en la comunidad y generan como consecuencia mayor presencia permanente de mujeres y niños.
Dado que no se cuenta con los niveles iniciales de alfabetización (jardín maternal-jardín de infantes), las mujeres tampoco realizan trabajos fueras de sus hogares o de su entorno inmediato. Asimismo, la distancia a los centros urbanos vecinos y las dificultades de acceso debido a la falta de frecuencia e infraestructura del transporte público imposibilitan que los niños, sobre todo de nivel primario, puedan asistir a clases. En los casos en que logran hacerlo, generalmente no es posible sostener en el tiempo la asiduidad de las clases. Por ejemplo, la asistencia irregular es la situación más frecuente.

En un contexto de madres semi o no alfabetizadas y niños semi o no alfabetizados, el crecimiento intelectual-letrado no resulta fácil, dado que el factor clave de interacción no puede lograrse.

Madres y abuelas, ávidas de conocimiento, fueron los actores centrales de este proyecto.

Es complejo describir esta necesidad sin caer en el vicio del juicio cultural. ¿Cuál es el lugar del conocimiento letrado en esta comunidad? ¿Cómo conciben la cultura letrada sus habitantes? $\mathrm{Y}$, fundamentalmente, ¿cómo detectar las necesidades desde una voz silenciada y diferente? ¿Cómo atender un pensamiento distinto sin juzgarlo desde la propia concepción? Éstos fueron los desafíos axiales por los que atravesó el equipo de voluntarios.

\section{Contexto y actores 2}

Un grupo de seis voluntarios trabajaron durante todo un año para tratar de acercar(se) respuestas a los interrogantes anteriores. Coordinados por un especialista en lenguas aborígenes, con acceso al campo mediante metodología etnográfica, tuvieron como consigna inicial visitar la comunidad, libres de expectativas. Consigna difícil para un joven próximo a egresar y decidido a cambiar el mundo rápidamente.

Los voluntarios trabajaron previamente desde diferentes cátedras universitarias nociones clave para llevar adelante este tipo de actividades. Cabe destacar en este sentido la importancia de la discusión respecto de la noción de "cultura", trabajo profundizado
1) Agradecemos especialmente a la Prof. Dra. Adriana Gonzalo por invitarnos a formar parte de esta propuesta y por confiar en nuestro trabajo. 2) Quinta: huerta, sembradío de verduras y hortaliza. 
desde la cátedra Sociología de la Cultura (materia optativa del plan de Profesorado en Letras) y que desde la propuesta de Raymond Williams permite poner en abismo la línea difusa que divide el prejuicio del reconocimiento. Así, la actividad de extensión puso de manifiesto para los voluntarios que no resulta igualmente "cómodo" interpelar el concepto a actuarlo concretamente en territorio. Por otro lado, desde la cátedra de Didácticas de la Lengua y la Literatura se recupera tal noción de "cultura" y se transpola a casos concretos y focalizados. Este ejercicio, si bien visualiza el medio, no es más que un artefacto ficticio que se acerca, pero no suplanta la actuación concreta en el medio. Desde esta misma cátedra nociones tales como "buena enseñanza" (Fenstermacher, 1995) y "obstáculo epistemológico" (Camilloni, 1997) resultaron centrales para la toma de decisiones vinculadas con los contenidos a enseñar y el método adoptado (ver Complejidades 2).

Los medios económicos que permitieron solventar los gastos del desplazamiento desde la ciudad de Santa Fe hasta la comunidad destino de la actividad, vinieron de la mano de un plan de alfabetización no formal que impulsaba el método "Yo, sí puedo". Este método, originado en Cuba y diseñado especialmente para la alfabetización de adultos, es de alto impacto en diferentes lugares del mundo. El método se direcciona desde lo conocido hacia lo nuevo; sobre el supuesto de que los números son un conocimiento adquirido para el mundo adulto, los números conducen hacia el conocimiento de las letras. Involucra dos actores claves además del propio sujeto a alfabetizar: el facilitador y los medios audiovisuales. El facilitador es el vínculo entre la clase audiovisual y el participante, desempeña una función importante en lo que respecta al trabajo de sensibilización y el monitoreo del aprendizaje. El material docente implicado en este método consta de una cartilla, un manual y 17 videos con 65 clases.

\section{Complejidades 1}

La distribución de la población de Campo San José, está claramente diferenciada en dos grupos (no nucleados territorialmente). Esta división, fruto de las lógicas sociales y de la diversidad y libertad de pensamiento, impactó en la organización del equipo, obligándolo a subdividirse. De esta manera, se organizó un equipo de voluntarios por grupo a alfabetizar (por ejemplo, dos grupos de tres personas, uno en cada sector de la comunidad). Este hecho obligó a duplicar los esfuerzos, repetir las clases y controlar con mayor rigor el avance de cada grupo.

El objetivo del proyecto y del equipo de voluntarios apuntó a la alfabetización inicial de adultos de la comunidad sin conocimientos previos o con experiencias escolares mínimas. Con claros propósitos de interrelación, aprendizaje y reconocimientos de las diferencias culturales, se abordó el trabajo desde una metodología de alfabetización que fue mutando y adaptándose a las necesidades del medio. El objetivo concreto, planteado desde el equipo, fue lograr avances básicos en la escritura, en la lectura y la posibilidad de rubricar la propia identidad (el propio yo). El desafío consistió en readaptar las expectativas y sobrellevar los obstáculos sociales y organizativos.

\section{Complejidades 2}

La lógica de trabajo del método "Yo, sí puedo" fue abandonándose gradualmente por diferentes motivos. La falta de infraestructura (específicamente el acceso a la energía eléctrica) dificultó el trabajo con los videos, disparadores de las clases. Este momento inicial de la clase, en el que el método prevé la visualización de un video, fue reemplazado por actividades de tipo lúdicas que apuntaron a atraer la atención hacia lo que, parafraseando a Andrueto (2008), podríamos llamar el inicio de un lector pleno. Así, a través del dibujo y de la música se atendió inicialmente a generar un vínculo con el lápiz y el papel. En esta instancia también, el equipo de letras (juego de letras en imprenta mayúscula pintadas sobre maderas que permiten la combinación para escribir palabras al modo de un rompecabezas) fue central para iniciar el proceso.

Por otro lado, quienes asistían a las clases tenían un conocimiento mucho más avanzado en lo relacionado con los números. Esto, que se creyó un beneficio en un primer momento, se volvió contraproducente en el sentido de que este método de alfabetización vincula números con letras de acuerdo con la siguiente lógica: los números se presentan de manera sucesiva mientras que las letras se incorporan por grado de dificultad.

A raíz de ello, muchas veces las clases no avanzaban no porque los asistentes no supieran los números, tampoco porque no recordasen las letras dadas, sino que el problema radicaba en la relación número-letra; los asistentes no recordaban la correlación.

\section{Complejidades 3}

El equipo debió enfrentar además dificultades totalmente fuera de su alcance. Las inclemencias del tiempo hicieron, en muchas oportunidades, distanciar los encuentros, alteraron la continuidad de las clases e impactaron de manera directa en los logros de la alfabetización.

La falta de infraestructura imposibilitó el dictado de las clases los días de lluvia o llovizna, esto así porque en uno de los sectores las clases se dictaban al aire libre, sumado a lo cual no era posible el acceso en días en los que persistía mucho el barro. En el otro sector sí se contaba con una sala de usos múltiples disponible 


\title{
66
}

\author{
¿Cuál es el lugar del conocimiento letrado \\ en esta comunidad? ¿Cómo conciben la \\ cultura letrada sus habitantes? ¿cómo \\ detectar las necesidades desde una voz \\ silenciada y diferente?
}

generalmente para esta actividad y su ubicación era más accesible. No obstante esto, se tomó la decisión de desfasar lo menos posible el trabajo del equipo completo.

La continuidad en el tiempo, con una frecuencia sostenida de encuentros, fue el principal factor para garantizar los resultados que se buscaban. El continuo retroceso para recordar los contenidos previamente presentados, retomarlos y afianzarlos se volvió en más de un momento, si bien una necesidad, también un punto de ahogo.

\section{Decisiones centrales}

Las primeras decisiones centrales que debieron tomarse fueron aquellas relacionadas con la forma de organización. Las madres y abuelas que asistían a las clases lo hacían llevando a los niños de la casa. De ese modo, se contaba con asistencia nutrida pero heterogénea. Los niños demandaban la atención de madres, abuelas y voluntarios al punto de obstaculizar e incluso impedir el desarrollo de la clase. A raíz de ello, la primera decisión central que se adoptó fue la de subdividir nuevamente a los voluntarios. En cada grupo de tres personas se reasignaron tareas. Así, un voluntario se hizo cargo de los niños, mientras que los otros dos llevaron adelante las clases.

La segunda decisión central que se adoptó fue referente al método de alfabetización. El ejercicio concreto en la práctica diaria permitió valorizar los enfoques de alfabetización en función de las particularidades del contexto de trabajo. Esto hizo posible tomar decisiones basadas en los conocimientos teóricos sobre los métodos, en consonancia con las respuestas de los actores involucrados. La reflexión sobre los métodos alternativos de alfabetización está ausente en la currícula del Profesorado de Letras. Claramente éste resultó ser el mayor obstáculo pedagógico
(Bachelard, 1948) al que los voluntarios debieron enfrentarse. La carrera supone que sus alumnos serán formadores de sujetos alfabetizados, y parte de ese punto para formarlos. No es nuestro objetivo aquí exponer nuestra opinión sobre esto, simplemente lo destacamos porque ubica a los voluntarios en una clara posición de desventaja conceptual que debieron reponer en pleno ejercicio en territorio de la mano de los coordinadores, expertos en el área. Con un conocimiento pleno de la situación, se acordó modificar la presentación de las clases corriéndose de la cartilla dada por "Yo, sí puedo" para proponer ejercicios que el mismo equipo elaboró en función de la propia práctica y de la lectura de materiales tales como La gramática de la fantasía de Gianni Rodari y, fundamentalmente, del Programa Nacional de Alfabetización y Educación Básica para Jóvenes y Adultos de Olinda Montenegro con ilustraciones de Roberto Fontanarrosa. Sobre la base de estos materiales se diseñaron nuevas clases que atendieron al ritmo y conocimientos previos de los asistentes. Estas nuevas actividades se basaron fundamentalmente en el método de alfabetización fonológico. Apelando a la conciencia fonológica, se presentó de a una letra por vez, recurriendo a mayúsculas y minúsculas imprenta, para luego lograr la combinación silábica que permitiera el paso a la palabra.

Para la organización del grupo de voluntarios y el seguimiento personalizado de cada uno de los adultos asistentes a las clases se elaboró una planilla en formato Excel de acceso abierto on-line para los integrantes del equipo a través de la aplicación Dropbox. De esta manera, todos los voluntarios tenían acceso a la hoja de ruta de cada alumno para atender a sus dificultades y potencialidades. Esta herramienta fue central, además, para evaluar los casos de permanencia y abandono.

La planilla de seguimiento grupal presentó el siguiente formato: 


\begin{tabular}{|c|c|c|c|c|c|c|c|c|}
\hline Clase & Fecha & \# de asistentes & \# de actividad & Logros & Dificultades & Tarea pendiente & Observaciones & Facilitador \\
\hline 1 & $5 /$ marzo & 14 & Toda la clase & $\begin{array}{l}\text { Seguían la clase } \\
\text { con mucho } \\
\text { interés. Se pudo } \\
\text { avanzar hasta la } \\
\text { letra "i". }\end{array}$ & $\begin{array}{l}\text { 1- Hay una señora } \\
\text { mayor (María), que de } \\
\text { lejos no ve, y se olvidó } \\
\text { sus anteojos. Va a un } \\
\text { ritmo más lento que } \\
\text { el resto de la clase, es } \\
\text { necesario esperarla. } \\
\text { 2- Hay una chica } \\
\text { (Teresa) que fue } \\
\text { hasta 3er año de la } \\
\text { secundaria y quiere } \\
\text { recordar matemática, } \\
\text { tiene interés en } \\
\text { terminar la escuela. }\end{array}$ & $\begin{array}{l}\text { Llevaron para realizar } \\
\text { el ejercicio de las } \\
\text { líneas de puntos, se } \\
\text { llevaron los cuadernos } \\
\text { y su respectivo lápiz. }\end{array}$ & $\begin{array}{l}\text { Hay muchos chicos } \\
\text { chiquitos. Es necesario } \\
\text { sacarlos a jugar o } \\
\text { realizar otra actividad } \\
\text { para que dejen trabajar } \\
\text { a sus mamás. A se } \\
\text { quedó dictando la clase } \\
\text { y B jugó con los chicos. } \\
\text { Se recomienda la } \\
\text { próxima semana poner } \\
\text { una mesa afuera } \\
\text { para que los chicos } \\
\text { hagan sus tareas, } \\
\text { o llevar juegos para } \\
\text { entretenerlos juna } \\
\text { PELOTA SERÍA IDEAL! } \\
\text { ¡Porque la mayoría son } \\
\text { varones!!! }\end{array}$ & $\mathrm{B}$ \\
\hline
\end{tabular}

Esta planilla permitió un trabajo prolijo y controlado sobre cada uno de los asistentes. Así, en la columna 1 se ingresó el número de la clase, en la 2 la fecha y en la 3 la cantidad de asistentes a dicha clase. En la columna 4 se consignó el número de la actividad hasta la cual se desarrolló esa clase. En la columna 5, los logros conseguidos y, en la 6 , las dificultades que se presentaron (generales y/o individuales). En la columna 7 se indicaron las actividades que quedaban pendientes de una clase para la otra, y en la columna 8 se consignó toda la información que los voluntarios consideraban relevante para mejorar el encuentro siguiente. Por último, en la columna final se indicó el nombre de quien completó la planilla, de manera que ante cualquier duda, los demás miembros del equipo pudieran contactarse con él. Esta planilla grupal se complementó con una planilla de seguimiento individual, la cual presentó el siguiente formato:
La planilla de seguimiento individual permitió el control de los avances y las dificultades de los asistentes, su grado de compromiso y sus actitudes ante las propuestas.

Estas herramientas fueron centrales para la organización del grupo, el seguimiento de los asistentes, el avance de la propuesta y el ejercicio de la propia práctica. Esto considerando especialmente la falta de continuidad en la asistencia y la optimización del tiempo de los voluntarios.

\begin{tabular}{|c|c|c|c|c|c|c|c|c|}
\hline Apellido & Nombre & Grupo & Estado inicial & Clase 1 & Observaciones & Facilitador & Clase 2 & $\ldots$ \\
\hline$X X X$ & YYY & Oeste & $\begin{array}{l}\text { Lee, no escribe } \\
\text { (fue hasta } 3^{\circ} \\
\text { grado) }\end{array}$ & Asistió & $\begin{array}{l}\text { Tiene vergüenza de } \\
\text { participar pero sabe } \\
\text { leer. Le cuesta escribir. }\end{array}$ & $A$ & Asistió & \\
\hline
\end{tabular}




\section{Desafíos y buenas prácticas}

Los voluntarios obtuvieron importantes logros en función de la superación de los obstáculos pedagógicos (mencionados anteriormente). Igualmente, lograron otorgar sentido a una práctica analizada conceptualmente en las aulas universitarias y discutidas en espacios de intercambio pero jamás actuadas en territorio, visibilizando así concretamente las dimensiones del desafío. De tal manera el empoderamiento de los asistentes en relación con su proceso de alfabetización fue de la mano del empoderamiento de los voluntarios en relación con su ejercicio pedagógico y social. A su vez, hizo posible comprender la necesidad de un plan lo suficientemente flexible como para permitir las fluctuaciones y mutaciones que el medio exija. El plan se adecua al medio y no a la inversa para estos casos.

También resultó evidente que el proceso de alfabetización de los adultos implica, al igual que para los niños, la necesidad de sostener acciones en el tiempo de manera continua y gradual. La concentración de actividades en un tiempo breve en períodos discontinuos no conduce a los objetivos esperados. Dadas las características del contexto y los rasgos supuestos (De Alba, 1994), clases breves sucesivas dieron mejores resultados.

Respecto de los actores, los asistentes lograron la escritura de su nombre y apellido, y la escritura de oraciones simples. El entusiasmo de los asistentes fluctuó de acuerdo, en la mayoría de los casos, con los demás problemas y urgencias que atravesaban a la comunidad en el día a día (reclamos, quejas, marchas,

inundaciones...).

La motivación de quien concurre a las clases no es sostenida y permanente. Los voluntarios tuvieron que poder afrontar este hecho que no está dentro de su campo de expectativas. El diseño de acciones de reatracción fue un desafío en sí mismo. Comprender que la concepción y el interés por el conocimiento no es una ni es única, fue otro gran desafío. Hacer carne el hecho de que la propia concepción sobre el conocimiento no es necesariamente la concepción correcta desde la cual se pueda juzgar el pensamiento de los demás fue el mayor de los desafíos.

Los voluntarios pudieron acceder a una realidad distinta y hasta el momento ajena, que les posibilitó revisar barreras sociales y culturales tanto en lo referente a los discursos de discriminación como, por supuesto, a aquellos discursos románticos casi congelados en tiempos pretéritos en los que se lee el ser aborigen como una marca de museo. Pudieron además conciliar el trabajo de extensión con el nivel de expectativa, vivenciar y compartir la pobreza material, diseñar acciones para suplir las faltasy sostener, aunque no sin vaivenes el entusiasmo mismo.

Sumado a esto, la problemática de la alfabetización básica y, más aún, de la alfabetización de adultos que, como se mencionó, está ausente en la currícula del Profesorado en Letras, supone un nivel de ejercicio docente al cual los demás actores acceden una vez lograda la alfabetización básica.
Así entonces, movidos por la curiosidad, la necesidad de acción y el autodesafío pusieron a prueba su capacidad de adaptación al medio de trabajo para acercar a sus conciudadanos un derecho básico para cualquier persona: saber leer y escribir.

En un claro ejercicio de problematización y resignificación de las prácticas de enseñanza, esta microintervención generó sin lugar a dudas modificaciones en las concepciones de mundo, lo cual no es otra cosa más que aprendizaje. En palabras de uno de los voluntarios (informe final):

"Una de las mayores frustraciones fue llegar a la comunidad y que no asista nadie a las clases por mirar programas de televisión, motivo que me parece injustificable. Sin embargo, uno no siempre puede luchar contra las ideologías y hábitos, por lo que, con el paso del tiempo, mis enojos y frustraciones primeros fueron apaciguándose".

Se aprendió de, desde y con el otro.

\section{Referencias bibliográficas}

Andruetto, M.T. (2008). Escribir. Recuperado de: http://www.ffyh.unc.edu.ar/ programas/propale/andruetto.htm (25/06/2016).

Bachelard, G. (1980 [1948]). La formación del espiritu cientifico. México: Siglo XXI Editores.

Camilloni, A. (1997). Los obstáculos epistemológicos en la enseñanza. Barcelona: Gedisa.

De Alba, A. (1994). Curriculum: crisis, mito y perspectivas. México: UNAM.

Fenstermacher, G. (1995). Tres aspectos de la filosofía de la investigación sobre la enseñanza. En Wittrock, M., La investigación de la enseñanza I. Enfoques teorías y métodos. Buenos Aires: Paidós.

Gerbaudo A. (2016). Politicas de exhumación. Las clases de los críticos en la universidad argentina de la posdictadura (1984-1986). Los Polvorines-Santa Fe: UNGS-UNL.

Montenegro, O. (2004). Programa Nacional de Alfabetización y Educación Básica para Jóvenes y Adultos. Buenos Aires: Ministerio de Educación de la Nación, Organización de Estados Iberoamericanos.

Rodari, G. (1983). La gramática de la fantasía. Barcelona: Argos Vergara. 